NASA Technical Memorandum 105588

ICOMP-92-06; CMOTT-92-03

\title{
Renormalization Group Analysis of the Reynolds Stress \\ Transport Equation
}

R. Rubinstein and J.M. Barton

Sverdrup Technology, Inc.

Lewis Research Center Group

Brook Park, Ohio

and Institute for Computational Mechanics in Propulsion

and Center for Modeling of Turbulence and Transition

Lewis Research Center

Cleveland, Ohio

March 1992

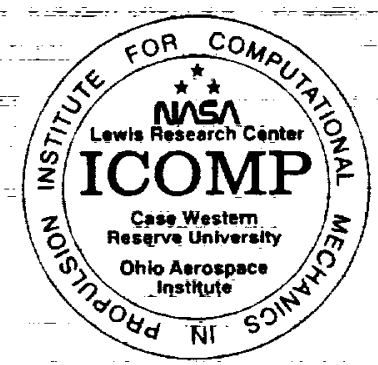




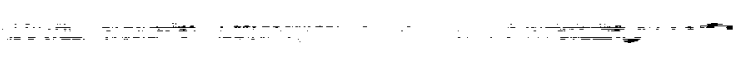




\title{
Renormalization Group Analysis of the Reynolds Stress \\ Transport Equation
}

\author{
R. Rubinstein and J.M. Barton \\ Sverdrup Technology, Inc. \\ Lewis Research Center Group \\ Brook Park, Ohio 44142 \\ and Institute for Computational Mechanics in Propulsion \\ and Center for Modeling of Turbulence and Transition* \\ Lewis Research Center \\ Cleveland, Ohio 44135
}

\begin{abstract}
The pressure-velocity correlation and return to isotropy term in the Reynolds stress transport equation are analyzed using the Yakhot-Orszag renormalization group. The perturlbation series for the relevant correlations, evaluated to lowest order in the $\epsilon$-cxpansion of the Yakhot-Orszag theory, are infinite series in tensor product powcrs of the mean vc$\approx$ locity gradient and its transpose. Formal lowest order Padé approximations to the sums of these series produce a fast pressure strain model of the form proposed by Launder, Reece, and Rodi, and a return to isotropy model of the form proposed by Rotta. In both cases, the model constants are computed theoretically. The predicted Reynolds stress ratios in simple shear flows are evaluated and compared with experimental data. The possibility is discussed of deriving higher order nonlinear models by approximating the sums more accurately.
\end{abstract}

${ }^{*}$ Work funded under NASA Cooperative Agreement NCC3-233. ICOMP Program Director, Louis A. Povinelli. 


\section{Introduction}

The modelling of the pressure correlation and return to isotropy term in the Reynolds stress transport equation remains controversial. ${ }^{1,2,3}$ Models will be derived here using the Yakhot-Orszag renormalization group ${ }^{4}$ partially along the lines of our previous work ${ }^{5}$. The result is a model for the fast pressure-strain term of the form proposed by Launder, Reece and Rodi ${ }^{6}$ (LRR) and a model for return to isotropy of the form proposed by Rotta ${ }^{7}$ with theoretically computed constants in good agreement with accepted values. As usual in investigations of this sort, the priority of Yoshizawa in deriving a pressure strain model analytically ${ }^{8}$ must be noted.

The analysis requires some new ideas in renormalization group theory recently introduced by Yakhot et $a^{9}$. As Yakhot et $a^{9}$ emphasize, the application of the renormalization group mode elimination formalism to shear flow creates a double perturbation series in powers of $\epsilon$, the parameter of the isotropic theory, and in powers of a dimensionless strain rate, $\eta=S K / \varepsilon$, where $K$ denotes the turbulence kinetic energy, $\varepsilon$ denotes the dissipation rate, and $S$ is a measure of the mean strain: in Ref. $9, S^{2}=\left(\frac{\partial U_{m}}{\partial x_{n}}+\frac{\partial U_{n}}{\partial x_{m}}\right) \frac{\partial U_{m}}{\partial x_{n}}$. The present analysis also leads to double expansions of this type, with the powers $S^{n}$ replaced by tensors $\mathbf{S}^{(n)}$ homogeneous of degree $n$ in the mean velocity gradient $\nabla U$ and its transpose. It will be convenient to retain the terminology of Ref. 9 and call this expansion the $\eta$-expansion; when the distinction is pertinent, the expansion of Ref. 9 will be called a scalar $\eta$-expansion.

The heuristic program of evaluating all scalar amplitudes to lowest order in $\epsilon$ has proven successful in the past: apparently, the $\epsilon$-expansion is an asymptotic series with sum given very nearly by its first term ${ }^{10}$. Unfortunately, there is no analogous basis for 
truncating the $\eta$-expansion. There are fundamental reasons for this distinction between these expansions. The present $\eta$-expansion is tensorial: successively higher order terms do not introduce merely numerical corrections, but increasingly complex asymmetries into the theory. Truncation therefore imposes a possibly inappropriate symmetry or other constraint on the model. Thus, in Ref. 5 the $\eta$-expansion for the Reynolds stress $\tau$ was truncated at second order as suggested by previous work of Yoshizawa ${ }^{11}$ and Speziale ${ }^{12}$. Although this type of modelling permits unequal normal stresses in a simple shear flow, it is not maximally asymmetric: for example, in a flow with mean velocity components $U_{i}\left(x_{1}, x_{2}\right)$, a cubic model including a term $\tau \sim \nabla U^{2} \nabla U^{T}+\nabla U \nabla U^{T 2}$ would permit nonzero $\tau_{23}$ in the presence of vanishing $\partial U_{2} / \partial x_{3}$, an effect which cannot be ruled out in advance.

Although generalizations ${ }^{\mathbf{1 3}}$ of the Cayley-Hamilton Theorem limit the number of independent tensors $\mathbf{S}^{(n)}$, anisotropy and asymmetry cannot exist at all without some terms of higher order in $\eta$; indeed, truncation at lowest order in $\eta$ just produces a theory of isotropic turbulence. But the series truncated at any higher order can be unsatisfactory in flow regions in which some components $\left(\frac{\partial U_{i}}{\partial x_{j}}\right) K / \varepsilon$ are large. In such regions, the truncated series is dominated by its highest order terms. For the quadratic stress models of Refs. 5, 11,12 , this domination can produce negative normal stresses in the buffer layers of wall bounded flows. Increasing the order of truncation obviously exacerbates this problem.

It follows that finite truncation of the $\eta$-expansion is theoretically unsatisfactory. Yakhot et $\mathrm{al}^{9}$ therefore propose that this expansion must be summed, even if only approximately, and have suggested a prototype summation in a different context. It should be noted that the same issues arise naturally in Yoshizawa's formalism, which also generates infinite series in the mean velocity gradients (and in other quantities as well) for 
correlations of interest in turbulence modeling. Yoshizawa has concluded independently that summation of this series is essential and has also derived a Reynolds stress transport model by introducing such summations ${ }^{14}$.

In this paper, the perturbation series which the Yakhot-Orszag renormalization group generates for the correlation

$$
\Pi_{i j}=-\left\langle u_{i} \frac{\partial p}{\partial x_{j}}+u_{j} \frac{\partial p}{\partial x_{i}}\right\rangle
$$

is summed by a low order Padé approximation. Coefficients are evaluated to lowest order in the $\epsilon$ expansion, but the summation includes effects of all orders in $\eta$. The result is essentially identical to the "model 1" proposed by Launder, Reece, and Rodi ${ }^{6}$. An entirely analogous treatment of return to isotropy yields a model of the form proposed by Rotta ${ }^{7}$. Combining these models leads to a preliminary Reynolds stress transport model. The problem of closing the Reynolds stress diffusion terms is addressed. This problem also leads to an infinite sum.

While it is encouraging that renormalization group methods can be used to derive familiar models, the goal of this investigation is not limited to providing theoretical justification for the LRR and Rotta models, which although widely applied are nevertheless deficient in several well-documented respects ${ }^{1,2,3}$. Instead, renormalization group methods together with approximate summation of the $\eta$-expansion can be used to derive higher order and nonlinear corrections to these models in a systematic fashion. Explicit development of such models is left to future investigations.

\section{Analysis of the Pressure Correlation}


The analysis will follow Yakhot and Orszag's derivation of turbulence transport models by renormalization group methods ${ }^{4}$. The equation for velocity products is

$$
\frac{\partial}{\partial t} u_{i} u_{j}+u_{p}\left(u_{i} \frac{\partial u_{j}}{\partial x_{p}}+u_{j} \frac{\partial u_{i}}{\partial x_{p}}\right)=-\left(u_{i} \frac{\partial p}{\partial x_{j}}+u_{j} \frac{\partial p}{\partial x_{i}}\right)+\nu_{0} \nabla^{2} u_{i} u_{j}-2 \nu_{0} \frac{\partial u_{i}}{\partial x_{p}} \frac{\partial u_{j}}{\partial x_{p}}
$$

where $\nu_{0}$ denotes the kinematic viscosity. The product $-\left(u_{i} \partial p / \partial x_{j}+u_{j} \partial p / \partial x_{i}\right)$ on the right side of Eq. (2) will become the correlation $\Pi_{i j}$ defined by Eq. (1) following elimination of all fluctuating modes. Its Fourier transform is

$$
\begin{aligned}
& \int u_{j}(\hat{k}-\hat{q}) i q_{i} q^{-2}\left(q_{p}-Q_{p}\right) u_{q}(\hat{q}-\hat{Q}) Q_{q} u_{p}(\hat{Q}) d \hat{q} d \hat{Q} /(2 \pi)^{2 d+2} \\
+ & \int u_{i}(\hat{k}-\hat{q}) i q_{j} q^{-2}\left(q_{p}-Q_{p}\right) u_{q}(\hat{q}-\hat{Q}) Q_{q} u_{p}(\hat{Q}) d \hat{q} d \hat{Q} /(2 \pi)^{2 d+2}
\end{aligned}
$$

Here the standard notation

$$
\hat{k}=(\omega, \mathbf{k}) \quad k^{2}=\mathbf{k} \cdot \mathbf{k}
$$

is used and $d=3$ is the number of space dimensions. Introduce an ultraviolet cutoff $\Lambda_{d}$ of the order of the inverse Kolmogorov scale $\left(\nu_{0}^{3} / \varepsilon\right)^{1 / 4}$; only inertial range scales with $k \leq \Lambda_{d}$ will be treated explicitly. Introducing a parameter $r$ initially near zero, partition wavenumber space $0 \leq k \leq \Lambda_{d}$ into the two intervals $0 \leq k \leq \Lambda_{d} e^{-r}$ and $\Lambda_{d} e^{-r} \leq k \leq \Lambda_{d}$. Denote velocity components with wavevectors in the first interval by the superscript < and those in the second by >. Introducing this decomposition into Eq. (3) produces eight terms; however, as in analogous calculations in Ref. 4, only three will contribute at the lowest order in $\epsilon$ : 


$$
\begin{aligned}
I & =\int u_{j}^{>}(\hat{k}-\hat{q}) i q_{i} q^{-2}\left(q_{p}-Q_{p}\right) u_{q}^{>}(\hat{q}-\hat{Q}) Q_{q} u_{p}^{<}(\hat{Q}) d \hat{q} d \hat{Q} /(2 \pi)^{2 d+2} \\
I I & =\int u_{j}^{>}(\hat{k}-\hat{q}) i q_{i} q^{-2}\left(q_{p}-Q_{p}\right) u_{q}^{<}(\hat{q}-\hat{Q}) Q_{q} u_{p}^{>}(\hat{Q}) d \hat{q} d \hat{Q} /(2 \pi)^{2 d+2} \\
I I I & =\int u_{j}^{<}(\hat{k}-\hat{q}) i q_{i} q^{-2}\left(q_{p}-Q_{p}\right) u_{q}^{>}(\hat{q}-\hat{Q}) Q_{q} u_{p}^{>}(\hat{Q}) d \hat{q} d \hat{Q} /(2 \pi)^{2 d+2}
\end{aligned}
$$

The $>$ modes are to be eliminated from these expressions by iterated use of the randomly forced Navier Stokes equations

$$
\left(-i \omega+\nu_{0} k^{2}\right) u_{i}(\hat{k})=-\frac{i}{2} \lambda_{0} P_{i m n}(\mathbf{k}) \int u_{m}(\hat{k}-\hat{q}) u_{m}(\hat{q}) d \hat{q} /(2 \pi)^{d+1}+f_{i}
$$

where

$$
\begin{aligned}
P_{i m n}(\mathbf{k}) & =k_{m} P_{i n}(\mathbf{k})+k_{n} P_{i m}(\mathbf{k}) \\
P_{i n}(\mathbf{k}) & =\delta_{i n}-k_{i} k_{n} / k^{2}
\end{aligned}
$$

and the Gaussian random force $f_{i}$ is defined by its correlation function

$$
\left\langle f_{i}(\hat{k}) f_{j}\left(\hat{k}^{\prime}\right)\right\rangle=2(2 \pi)^{d+1} D_{0} \delta\left(\omega+\omega^{\prime}\right) \delta\left(\mathbf{k}+\mathbf{k}^{\prime}\right) k^{-y}
$$

The choice $y=d$ generates a Kolmogorov inertial range. A detailed exposition of this procedure can be found in Ref. 4. It will suffice to note here that the result of the mode elimination is a series in powers of $u^{<}$and $\lambda_{0}$. At each order in $u^{<}$, perturbation theory will produce a finite number of types of terms with amplitudes given as series in $\lambda_{0}$. Under iterated mode elimination to the limit $r \rightarrow \infty$, the expansion in $\lambda_{0}$ proves to be an expansion in powers of $\epsilon=4+y-d$. This is the $\epsilon$-expansion of the Yakhot-Orszag theory. Previous experience ${ }^{4}$ and preliminary analysis ${ }^{10}$ suggest that the amplitudes are best evaluated at lowest order in $\epsilon$ with $\epsilon$ set to zero. This procedure will be followed here. 
Thus, the perturbation series will be written as

$$
\Pi=T_{0}+T_{1}+\cdots
$$

where $T_{n}$ is of order $n$ in $u^{<}$and all amplitudes are evaluated to lowest order in $\epsilon$. To lowest order in $\epsilon$ and $\mathbf{S} K / \epsilon$

$$
I I I=\int \delta(\mathbf{q}) q_{i} q_{p} q^{-2} Q_{q} u_{j}(\hat{k}) u_{q}(-\hat{Q}) u_{p}(\hat{Q}) d \hat{q} /(2 \pi)^{d+1} \equiv 0
$$

since incompressibility implies $Q_{q} u_{q} \equiv 0$. Now term $I I I$ is formally proportional to $q_{i} u_{j}$ and since only indices $i$ and $j$ are uncontracted, the combination $Q_{p} u_{p}$ or $Q_{q} u_{q}$ must occur at all orders. Accordingly, term $I I I$ vanishes identically.

Lowest order analysis of term $I$ gives

$$
\int q_{i} q_{p} q^{-2-y}\left\{|G(\hat{q})|^{2} P_{j q}(\mathbf{q}) 2 D_{o} d \hat{q} /(2 \pi)^{d+1}\right\} \cdot i k_{q} u_{p}(\hat{k})
$$

Adding the corresponding term from $I I$ and the result of $i j$ index interchange leads to

$$
T_{1}=\hat{T}_{1}\left(\frac{\partial U_{i}^{<}}{\partial x_{j}}+\frac{\partial U_{j}^{<}}{\partial x_{i}}\right)
$$

where $\hat{T}_{1}$ satisfies the recursion relation

$$
\frac{d \hat{T}_{1}}{d r}=\frac{6}{15} \frac{\mathcal{D}}{\nu \Lambda^{2}}
$$

In Eq. (5), $y$ has been set equal to $d$ to obtain Kolmogorov scaling and

$$
\mathcal{D}=2 D_{0} S_{d} /(2 \pi)^{d}
$$




$$
\Lambda=\Lambda_{d} e^{-r}
$$

where $S_{d}$ denotes the area of the $d$-dimensional sphere. Integrating the recursion relation (5) in the high Reynolds number asymptotic limit ${ }^{4} r \rightarrow \infty$,

$$
\hat{T}_{1}=\frac{2}{5} K
$$

Eqs. (4) and (6) give

$$
T_{1}=\frac{2}{5} K\left(\frac{\partial U_{i}}{\partial x_{j}}+\frac{\partial U_{j}}{\partial x_{i}}\right)
$$

in agreement with the analysis of Crow. ${ }^{16}$

At the next order in $\mathbf{S} K / \varepsilon$,

$$
\begin{aligned}
I= & \int q_{i} q_{p} q^{-2}\left\{P_{j r s}(-\mathbf{q}) G(-\hat{q})|G(\hat{q}-\hat{Q})|^{2} \times\right. \\
& \left.P_{q s}(\mathbf{Q}-\mathbf{q})|\mathbf{Q}-\mathbf{q}|^{-y} 2 D_{0} \cdot v_{r}(\hat{k}-\hat{Q})\right\} d \hat{q} /(2 \pi)^{d+1} \cdot i Q_{q} u_{p}(\hat{Q})+(i j)
\end{aligned}
$$

where $(i j)$ indicates the result of $i j$ index interchange in the previous term. Adding the contribution from $I I$,

$$
T_{2}=\hat{T}_{2} \frac{1}{105}\left[16\left(\frac{\partial u_{j}^{<}}{\partial x_{p}}+\frac{\partial u_{p}^{<}}{\partial x_{j}}\right) \frac{\partial u_{i}^{<}}{\partial x_{p}}+2\left(\frac{\partial u_{j}^{<}}{\partial x_{p}}+\frac{\partial u_{p}^{<}}{\partial x_{j}}\right) \frac{\partial u_{p}^{<}}{\partial x_{i}}\right]^{(0)}+(i j)
$$

where (0) indicates the deviatoric part: it is immediate that the $i j$ index contraction of Eq. (8) vanishes. The amplitude $\hat{T}_{2}$ satisfies

$$
\frac{d \hat{T}_{2}}{d r}=-\frac{\mathcal{D}}{4 \nu^{2} \Lambda^{4}}
$$

In the high Reynolds number limit, 


$$
\begin{aligned}
T_{2} & =-\frac{1}{105} \frac{1}{4} \frac{40}{3} \frac{3}{4} \nu\left[32\left(\frac{\partial U_{i}}{\partial x_{p}}+\frac{\partial U_{p}}{\partial x_{i}}\right) \frac{\partial U_{j}}{\partial x_{p}}+4\left(\frac{\partial U_{i}}{\partial x_{p}}+\frac{\partial U_{p}}{\partial x_{i}}\right) \frac{\partial U_{p}}{\partial x_{j}}\right]^{(0)}+(i j) \\
& =-\frac{1}{21} \nu\left[16\left(\frac{\partial U_{i}}{\partial x_{p}}+\frac{\partial U_{p}}{\partial x_{i}}\right) \frac{\partial U_{j}}{\partial x_{p}}+2\left(\frac{\partial U_{i}}{\partial x_{p}}+\frac{\partial U_{p}}{\partial x_{i}}\right) \frac{\partial U_{p}}{\partial x_{j}}\right]^{(0)}+(i j)
\end{aligned}
$$

The next order will produce a term $T_{3}$ containing cubic products of velocities $u^{<}$. In view of the form of the LRR model, it is reasonable to ask whether a term with only one gradient, proportional in the high Reynolds number limit to $\tau \nabla U$ might occur at this order. Such terms do occur, but they cancel. Evaluation of $T_{3}$ proves to require expansions of the projection operators to second order, leading instead to terms $\mathbf{S}^{(3)}$ homogeneous of degree three in the mean velocity gradient and its transpose. In general, the term $T_{n}$ of order $n$ has the form $\mathbf{S}^{(n)}(K / \varepsilon)^{n}$. As noted in the Introduction, it will be imperative to include effects of all orders in $\mathrm{S} K / \varepsilon$ in the model, but because the terms $T_{n}$ involve ever higher order derivatives of the transverse projection operators, they do not have an obvious law of formation. Therefore, an exact summation does not appear feasible.

It can be verified that the terms in braces in $T_{1}$ and $T_{2}$ are the lowest order terms in the expansion of the correlation spectrum tensor $E_{m n}$ with the property

$$
\overline{u_{m} u_{n}}=\int E_{m n}(\mathbf{q}) d \mathbf{q}
$$

and that this identification holds to all orders. Thus, perturbation theory gives the standard result

$$
\Pi_{i j}=\int q^{-2}\left[q_{i p} E_{j q}+q_{j p} E_{i q}\right] d \mathbf{q} \cdot \frac{\partial U_{p}}{\partial x_{q}}
$$


but with a series for the right hand side which can be evaluated explicitly to any finite order. In fact, in view of Eqs. (7) and (9),

$$
\begin{gathered}
\Pi_{i j}=\frac{2}{5} K\left(\frac{\partial U_{i}}{\partial x_{j}}+\frac{\partial U_{j}}{\partial x_{i}}\right) \\
-\frac{16}{21} \nu\left[\left(\frac{\partial U_{i}}{\partial x_{p}}+\frac{\partial U_{p}}{\partial x_{i}}\right) \frac{\partial U_{j}}{\partial x_{p}}+\left(\frac{\partial U_{j}}{\partial x_{p}}+\frac{\partial U_{p}}{\partial x_{j}}\right) \frac{\partial U_{i}}{\partial x_{p}}\right] \\
-\frac{2}{21} \nu\left[\left(\frac{\partial U_{i}}{\partial x_{p}}+\frac{\partial U_{p}}{\partial x_{i}}\right) \frac{\partial U_{p}}{\partial x_{j}}+\left(\frac{\partial U_{j}}{\partial x_{p}}+\frac{\partial U_{p}}{\partial x_{j}}\right) \frac{\partial U_{p}}{\partial x_{i}}\right]+\varepsilon \sum_{n \geq 3} \mathrm{~S}^{(n)}(K / \varepsilon)^{n}
\end{gathered}
$$

A simple approximate summation is obtained by introducing into Eq. (10) the perturbation series ${ }^{5}$ for ${\overline{u_{i} u_{j}}}^{(0)}$ in the form

$$
\nu\left(\frac{\partial U_{i}}{\partial x_{j}}+\frac{\partial U_{j}}{\partial x_{i}}\right)=-{\overline{u_{i} u_{j}}}^{(0)}+\sum_{n \geq 2} \mathbf{S}^{(n)}(K / \varepsilon)^{n}
$$

and dropping the quadratic terms. The resulting model,

$$
\begin{aligned}
\Pi_{i j}= & \frac{2}{5} K\left(\frac{\partial U_{i}}{\partial x_{j}}+\frac{\partial U_{j}}{\partial x_{i}}\right)+C_{\dot{\tau} 1}\left[{\overline{u_{i} u_{p}}}^{(0)} \frac{\partial U_{j}}{\partial x_{p}}+{\overline{u_{j} u_{p}}}^{(0)} \frac{\partial U_{i}}{\partial x_{p}}\right]^{(0)} \\
& +C_{\dot{\tau} 2}\left[{\overline{u_{i} u_{p}}}^{(0)} \frac{\partial U_{p}}{\partial x_{j}}+{\overline{u_{j} u_{p}}}^{(0)} \frac{\partial U_{p}}{\partial x_{i}}\right]^{(0)}
\end{aligned}
$$

with

$$
C_{\dot{r} 1}=\frac{16}{21}=.7619 \quad C_{\dot{r} 2}=\frac{2}{21}=.0952
$$

agrees with the perturbation series (10) to terms of order $\mathbf{S}^{(3)}$. However, unlike the explicit quadratic model which results from simply dropping the $O\left(\mathbf{S}^{(3)}\right)$ terms in Eq. (10), this model includes effects of all order in $\mathrm{S} K / \varepsilon$. The consequences of this fact will be discussed later. This type of summation has also been applied by Yoshizawa ${ }^{14}$. Eqs. (11) and (12) 
can be compared with Launder, Reece and Rodi's "model 1", Eq. (11) with the empirically adjusted constants

$$
C_{\dot{\tau} 1}=.7636 \quad C_{\dot{\tau} 2}=.1091
$$

The constants $C_{\dot{r} 1}$ and $C_{\dot{r} 2}$ are not chosen independently; instead, to insure certain consistency properties ${ }^{6,7}$, they are linear functions of a parameter $C_{2}$. The LRR model corresponds to the choice $C_{2}=.4$; Eqs. (11) and (12) correspond instead to the choice $C_{2}=8 / 21 \sim .36$

The approximate summation used to derive Eq. (11) can be systematically generalized to generate an infinite number of models for $\Pi_{i j}$. For example, suppose that the perturbation series for $\tau$ is introduced into the cubic terms in the perturbation series (10) instead of in the quadratic terms as above. This substitution will produce a model which can be written symbolically in the form

$$
\Pi \sim \mathbf{S}^{(1)}+\mathbf{S}^{(2)}+\tau\left(\mathbf{S}^{(1)^{\prime}}+\mathbf{S}^{(2)^{\prime}}\right)
$$

where $\tau \mathbf{S}^{(i)^{\prime}}$ denotes a sum of matrix products in all possible orders of $\tau$ and terms $\mathbf{S}^{(i)}$. The requirement that the original series agree to order $\mathbf{S}^{(4)}$ with the approximation when $\tau$ is replaced by its perturbation series determines this approximation uniquely.

There is obviously a strong formal resemblance between this approximation scheme and Padé approximation. They differ in the introduction of $\tau$ as an auxiliary quantity, but more fundamentally in the non-commutativity of all of the variables $\tau, \nabla U$, and $\nabla U^{T}$. It will be evident in Sect. IV that introduction of these approximations into a Reynolds 
stress transport equation will lead under suitable hypotheses to algebraic models for $\tau$ of the form

$$
\tau \sim K\left(\mathcal{S}^{(1)}+\ldots+\mathcal{S}^{(n)}\right)+\tau\left(\mathcal{S}^{(1)^{\prime}}+\ldots+\mathcal{S}^{(n)^{\prime}}\right)
$$

which formally express $\tau$ as a ratio of polynomials of degree $n$ in $\nabla U$ and $\nabla U^{T}$. It is noteworthy that the LRR model is the lowest order member of this series. Moreover, the analysis suggests that there is no unique optimal form for the closure of $\Pi$ in terms of $\tau, \nabla U$, and $\nabla U^{T}$; instead, there is a series of approximations of (presumably) increasing accuracy.

The LRR model has been criticized by Shih and Lumley ${ }^{2}$ because it fails to insure realizability at the limit of two component flow. Speziale ${ }^{1}$ has found different limitations when this model is applied to homogeneous shear flow. It is not clear whether a different summation procedure would lead to models which could answer these criticisms, perhaps of the forms proposed in Refs. 1 and 2. However, these references also indicate that what conditions a good model should satisfy is itself a somewhat controversial question. Accordingly, the agreement of this theory with a plausible and often used pressure strain model is encouraging.

\section{The Return to Isotropy Model}

The analytical description of return to isotropy is no less controversial than the modeling of the fast pressure strain term ${ }^{3}$. In the usual approach to turbulence modeling, in which correlations generated by Reynolds averaging are closed phenomenologically, this process is considered to result partly from the pressure correlation through a "slow" term 
independent of the mean flow, and partly from the deviatoric part of the dissipative correlation $\left\langle\nu_{0} \frac{\partial u_{i}}{\partial x_{p}} \frac{\partial u_{j}}{\partial x_{p}}\right\rangle$. From this viewpoint, the analysis in Sect. I is incomplete because it discloses only a term proportional to the mean velocity gradient, but no slow term. The return to isotropy will be derived here by renormalization group methods following a suggestion of Yakhot ${ }^{17}$.

From the renormalization group viewpoint, it is natural to investigate the return to isotropy, even independently of the stress transport equation, by writing the perturbation series for

$$
u_{i} \frac{\partial u_{j}}{\partial t}+u_{j} \frac{\partial u_{i}}{\partial t}=\int u_{i}(\hat{k}-\hat{q})(-i \omega) u_{j}(\hat{q}) d \hat{q}+(i j)
$$

This perturbation series differs from the perturbation series for the Reynolds stresses previously reported ${ }^{5}$ only in the occurence of an additional factor $-i \omega$ in all frequency integrals. It is therefore natural to identify the sum of this series as a functional of the Reynolds stresses which, like the slow term of turbulence modeling, is independent of the mean flow. By substituting the Navier-Stokes equations for the time derivatives in Eq. (14), one just recovers the equations of motion for velocity products, Eq. (2); thus, the quantity

$$
\Pi_{i j}^{\prime}=\left\langle u_{i} \frac{\partial u_{j}}{\partial t}+u_{j} \frac{\partial u_{i}}{\partial t}\right\rangle
$$

which results from eliminating all fluctuating modes from Eq. (14) contains contributions from the pressure correlation through terms containing the transverse projection operator and contributions from the dissipative correlation through the terms containing $\nu_{0}$.

By evaluating these terms in the form of Eq. (14) and insisting that the sum be independent of the mean flow, we are extracting properties which were not disclosed in the analysis of Sect. I. 
The analysis is straightforward. Only the deviatoric terms require attention because the part of the correlation proporational to $\delta_{i j}$ contributes to the transport equation for $K$ which has been analyzed by Yakhot and $\mathrm{Smith}^{15}$. The lowest order deviator appears at first order in $\eta$; to lowest order in $\epsilon$

$$
T_{1}=\hat{T}_{1}\left(\frac{\partial U_{i}}{\partial x_{j}}+\frac{\partial U_{j}}{\partial x_{i}}\right)
$$

where

$$
\frac{d \hat{T}_{1}}{d r}=\frac{1}{15} \frac{\mathcal{D}}{\nu \Lambda^{2}}
$$

In view of the form of the Rotta model, it is reasonable to seek terms at the next order proportional to $u_{i} u_{j}$. As in Sect. I, such terms do appear, but cancel exactly. This apparently ubiquitous cancellation was also obtained by Smith and Reynolds ${ }^{18}$ in an analysis of the $\varepsilon$ transport equation. Accordingly, the second order analysis in $\eta$ produces quadratic terms in the velocity gradients. Finite truncation of this series violates the requirement that return to isotropy be independent of the mean flow. Therefore, we must seek a reasonable approximate summation. The form of the lowest order term given in Eqs. (15) and (16) suggests

$$
\Pi_{i j}^{\prime}=\frac{\hat{T}_{1}}{\nu} \nu\left(\frac{\partial U_{i}}{\partial x_{j}}+\frac{\partial U_{j}}{\partial x_{i}}\right) \sim-\frac{\hat{T}_{1}}{\nu} \overline{u_{i} u_{j}}(0)
$$

Despite its triviality, this replacement does produce an approximate sum which agrees exactly with perturbation theory to lowest order. It therefore can be considered a type of Padé approximation. This lowest order summation yields 


$$
\Pi_{i j}^{\prime}=-Z{\overline{u_{i}}}_{u_{j}}^{(0)}
$$

where

$$
\frac{d Z}{d r}=\frac{1}{15} \frac{\mathcal{D}}{\nu^{2} \Lambda^{2}}
$$

At the infinite Reynolds number asymptotic limit, Eqs. (17) and (18) iterate to ${ }^{4}$

$$
\Pi_{i j}^{\prime}=-C_{R} \frac{\varepsilon}{K}{\overline{u_{i} u_{j}}}^{(0)}
$$

where, in the Yakhot-Orszag theory, $C_{R}=\mathcal{D} / \varepsilon \sim 1.6$. Equation (19) is therefore simply the standard Rotta model with Rotta constant $\sim 1.6$ in agreement with an earlier proposal of Yakhot ${ }^{17}$.

A preliminary discussion of higher order summation may be appropriate. By analyzing the spectral dynamics of the return to isotropy, Weinstock ${ }^{3}$ concluded that the shear and normal stresses relax at different rates. Although this behavior is obviously not accommodated by the Rotta model, it is consistent with the present theory: the perturbation series for $\Pi^{\prime}$ is obtained from the series for $\tau$ by multiplying the term of order $n$ by the factor $C_{n} \varepsilon / K$ for some constant $C_{n}$. The $C_{n}$ are all unequal; therefore, the Rotta model is not exact. Now comparison with the series for $\tau$ shows $^{5}$ that relaxation of the shear stress is governed by the linear term $\mathbf{S}^{(1)}$, whereas relaxation of the normal stresses is governed by the quadratic term $\mathbf{S}^{(2)}$. Since $C_{2} \neq C_{1}$, these stresses relax at different rates. The difference is suppressed in the Rotta model, which arose in the present formalism by replacing all of the $C_{n}$ by $C_{1}$. 


\section{Reynolds Stress Transport Models}

The renormalization group describes the effect of the universal small scales of turbulence on the large scales. Therefore, convection and production, which are determined entirely by the large scales, cannot be derived by the renormalization group and must be introduced instead by Reynolds averaging ${ }^{15}$. Combining the usual convection and production terms with the pressure correlation and return to isotropy models, Eqs. (11) and (19), gives the Reynolds stress transport equation

$$
\begin{aligned}
& \frac{\partial{\overline{u_{i} u_{j}}}^{(0)}}{\partial t}+U_{p} \frac{\partial{\overline{u_{i} u_{j}}}^{(0)}}{\partial x_{p}}=-\left(\overline{u_{i} u_{p}} \frac{\partial U_{j}}{\partial x_{p}}+{\overline{u_{j} u_{p}}}_{\frac{\partial U_{i}}{\partial x_{p}}}\right)^{(0)}-C_{R} \frac{\varepsilon}{K}{\overline{u_{i} u_{j}}}^{(0)} \\
& +\frac{2}{5} K\left(\frac{\partial U_{i}}{\partial x_{j}}+\frac{\partial U_{j}}{\partial x_{i}}\right)+C_{\dot{\tau} 1}\left({\overline{u_{i} u_{p}}}^{(0)} \frac{\partial U_{j}}{\partial x_{p}}+{\overline{u_{j} u_{p}}}^{(0)} \frac{\partial U_{i}}{\partial x_{p}}\right)^{(0)} \\
& +C_{i 2}\left({\overline{u_{i} u_{p}}}^{(0)} \frac{\partial U_{p}}{\partial x_{j}}+{\overline{u_{j} u_{p}}}^{(0)} \frac{\partial U_{p}}{\partial x_{i}}\right)^{(0)}+\operatorname{diff} .
\end{aligned}
$$

where "diff" denotes diffusion terms which will be discussed later. The predictions of models of the form (20) for homogeneous shear flow, in which the diffusion terms vanish, have been analyzed definitively by Speziale. ${ }^{19}$ It will be useful to generalize this analysis somewhat and consider any simple shear flow with exactly one nonvanishing mean velocity gradient component $S=\partial U_{1} / \partial x_{2}$, in which diffusion of all Reynolds stress components is negligible, and in which $\overline{u_{i} u_{j}} / K$ is constant. These are the conditions under which Rodi's algebraic models ${ }^{20}$ can be derived and include homogeneous shear flow as a special case. It follows from Eq. (20) that under these conditions,

$$
\left(\overline{u_{1} u_{2}} / K\right)^{2}=\frac{4}{15} \frac{P / \varepsilon}{C_{R}+P / \varepsilon-1}-\frac{2}{3}\left(\frac{P / \varepsilon}{C_{R}+P / \varepsilon-1}\right)^{2}\left[\left(C_{\dot{r} 1}-1\right)^{2}\right.
$$




$$
\begin{gathered}
\left.-4\left(C_{\dot{r} 1}-1\right) C_{\dot{\tau} 2}+C_{\dot{r} 2}^{2}\right] \\
{\overline{u_{1} u_{1}}}^{(0)} / K=\frac{P / \varepsilon}{C_{R}+P / \varepsilon-1}\left[-\frac{4}{3}\left(C_{\dot{r} 1}-1\right)+\frac{2}{3} C_{\dot{r} 2}\right] \\
-{\overline{u_{2} u_{2}}}^{(0)} / K=\frac{P / \varepsilon}{C_{R}+P / \varepsilon-1}\left[-\frac{2}{3}\left(C_{\dot{r} 1}-1\right)+\frac{4}{3} C_{\dot{r} 2}\right]
\end{gathered}
$$

where $P=-S \overline{u_{1} u_{2}}$ is production. Note in particular that the ratio

$$
-{\overline{u_{1} u_{1}}}^{(0)} /{\overline{u_{2} u_{2}}}^{(0)}=\frac{-\frac{4}{3}\left(C_{\dot{r} 1}-1\right)+\frac{2}{3} C_{\dot{r} 2}}{-\frac{2}{3}\left(C_{\dot{r} 1}-1\right)+\frac{4}{3} C_{\dot{\tau} 2}}
$$

is independent of both $P / \varepsilon$ and of the model constant $C_{R}$.

These results can be applied to model calibration. Rotta noted ${ }^{7}$ that certain consistency conditions require

$$
8 C_{\dot{+} 1}-C_{\dot{\tau} 2}=6
$$

In view of Eqs. (22) and (23), the model proposed here, which is defined by the values of $C_{\dot{r} 1}$ and $C_{\dot{r} 2}$ of Eq. (12) is the unique model of its form for which

$$
-{\overline{u_{1} u_{1}}}^{(0)} /{\overline{u_{2} u_{2}}}^{(0)}=4 / 3
$$

The validity of Eq. (24) can be assessed from the experimental data summarized in Table II. Other Reynolds stress ratios can also be readily evaluated. Substituting Eq. (23) into Eqs. (21)

$$
\begin{gathered}
{\overline{u_{1} u_{1}}}^{(0)} / K=C^{*}\left(4 C_{\dot{\tau} 1}-\frac{8}{3}\right) \\
-{\overline{u_{2} u_{2}}}^{(0)} / K=C^{*}\left(10 C_{\dot{\tau} 1}-\frac{22}{3}\right) \\
-{\overline{u_{3} u_{3}}}^{(0)} / K=C^{*}\left(-6 C_{\dot{\tau} 1}-\frac{14}{3}\right)
\end{gathered}
$$




$$
\left(\overline{u_{1} u_{2}} / K\right)^{2}=\frac{4}{15} C^{*}-\frac{2}{3}\left(C^{*}\right)^{2}\left(33 C_{\dot{+} 1}^{2}-42 C_{\dot{\tau} 1}+13\right)
$$

where

$$
C^{*}=\frac{P / \varepsilon}{C_{R}+P / \varepsilon-1}
$$

Simple shear flow data suggest that ${\overline{u_{1} u_{1}}}^{(0)} / K,-{\overline{u_{2} u_{2}}}^{(0)} / K$, and $-{\overline{u_{3} u_{3}}}^{(0)} / K$ are all positive. In view of Eq. (25), this forces $C_{\dot{r} 1}$ to be in the narrow interval

$$
\frac{11}{15} \leq C_{\dot{\tau} 1} \leq \frac{7}{9}
$$

from about .73 to .78 . Values of the Reynolds stress ratios for various values of $C_{\dot{\tau} 1}$ appear in Table I. No value of $C_{\dot{r} 1}$ gives entirely satisfactory values for all of the ratios; in particular, the ratio ${\overline{u_{1} u_{1}}}^{(0)} / K \sim .4$ in homogeneous shear flow cannot be obtained from a model of this form.

Phenomenological modelling has suggested numerous modifications of the original LRR form. The simplest modification retains the form of Eq. (11), but drops the constraint expressed by Eq. (23). Thus, the constants $C_{\dot{\tau} 1}$ and $C_{\dot{\tau} 2}$ are considered independent, as in Launder, Reece and Rodi's "model 2"6 , or in the model of Speziale, Sarkar, and Gatski" Justification of this step requires that the idea of modelling each individual correlation in the exact Reynolds stress transport equation be abandoned; instead, the model is proposed for the entire equation at once. A reasonable model of this type is Eq. (11) with the constants

$$
C_{\dot{r} 1}=\frac{43}{63} \quad C_{\dot{r} 2}=\frac{8}{63}
$$


chosen so that the Reynolds stress transport equation reduces to the nonlinear model of Ref. 5 when convection and diffusion of Reynolds stress are negligible. The values of the Reynolds stress ratios for this model in Table I are in excellent agreement with shear flow data, but the fundamental validity of such modelling is unclear. An alternative procedure is to explore the higher order summations described in Sect. I.

A point emphasized by both Speziale ${ }^{19}$ and Reynolds ${ }^{21}$ can be noted here, that while the elementary eddy viscosity formula and its nonlinear generalization ${ }^{5,11,12}$ applied to simple shear flow give

$$
\overline{u_{1} u_{2}} / K \sim S K / \varepsilon \quad{\overline{u_{i} u_{i}}}^{(0)} / K \sim(S K / \varepsilon)^{2}
$$

for any value of $S K / \varepsilon$, models of the form of Eq. (20) give (26) for moderate $S K / \varepsilon$, but

$$
\overline{u_{1} u_{2}} / K \sim \text { const. } \quad{\overline{u_{i} u_{i}}}^{(0)} / K \sim \text { const. }
$$

in the rapid distortion limit $S K / \varepsilon \rightarrow \infty$. The finite limit expressed by Eq. (27) also holds for algebraic Reynolds stress models derived following Rodi's original suggestion ${ }^{20}$, for example for the model proposed in Ref. 22,

$$
\nu_{T}=\frac{2}{15} \frac{K^{2} / \varepsilon}{C_{R}+(P / \varepsilon-1)}
$$

The behavior (26) is certainly incorrect when $S K / \varepsilon \rightarrow \infty$; this reflects the derivation, for example of the relation $\nu_{T} \sim K^{2} / \varepsilon$ from Kolmogorov scaling. This derivation assumes quasi-static spectral evolution in which the Kolmogorov spectrum instantaneously adjusts to local conditions in both space and time. The finite limit (27) at large $S K / \varepsilon$ can only 
occur if effects of all order in $S K / \varepsilon$ are included. No finite truncation of the $\eta$-expansion will have this behavior.

\section{Algebraic Reynolds Stress Models}

The approximation, due to $\operatorname{Rodi}^{20}$, of the Reynolds stress transport equation by an algebraic model under the conditions of semi-homogeneous flow (negligible diffusion of $\tau$ and $\tau / K$ approximately constant) takes the form

$$
\frac{P-\varepsilon}{K}{\overline{u_{i} u_{j}}}^{(0)}=-\left(\overline{u_{i} u_{p}} \frac{\partial U_{j}}{\partial x_{p}}+\overline{u_{j} u_{p}} \frac{\partial U_{i}}{\partial x_{p}}\right)^{(0)}+\Pi_{i j}^{\prime}+\Pi_{i j}
$$

where $\Pi$ and $\Pi^{\prime}$ depend on $\tau$ and $\nabla U$. Explicit solutions for $\tau$ can be obtained, at least in principle, for any such approximation ${ }^{23}$. Briefly, one introduces a basis for polynomials in $\nabla U$, and $\nabla U^{T}$. The basis contains 11 terms of homogeneity order $n \leq 5$. Writing $\tau$ as a sum of these terms with unknown coefficients and substituting in Eq. (29) leads to the explicit expression

$$
\tau / K=\sum H_{i}^{(m)} \mathbf{S}_{i}^{(n)}\left(\nabla U, \nabla U^{T}\right)
$$

where $H_{i}^{(m)}$ is a scalar function of $\nabla U$ and $\nabla U^{T}$ such that

$$
H_{i}^{(m)} \sim|\nabla U|^{m}
$$

when $|\nabla U| \rightarrow \infty$. The assumptions made on the approximate summations require $m+n=$ 0 ; thus, $\tau / K$ is bounded when $S K / \varepsilon \rightarrow \infty$. For example, the familiar eddy viscosity formula is replaced in Eq. (30) by a term 


$$
\tau \sim \frac{K^{2}}{\varepsilon} H^{(-1)}\left(\nabla U, \nabla U^{T}\right)\left(\nabla U+\nabla U^{T}\right)
$$

This is the type of eddy viscosity modification sought by Horiuti ${ }^{24}$, but with the boundedness property expressed by Eq. (27).

Pope observed ${ }^{23}$ that the coefficients $H^{(-n)}$ in Eq. (30) would certainly be intractably complex; although they could be explicitly exhibited by symbolic computation, the result would only pertain to the particular implicit equation for the Reynolds stresses assumed initially in Eq. (29). Therefore, it is equally reasonable just to postulate simple forms for the functions $H^{(-n)}$; for example, Eq. (28) suggests the possibility

$$
H^{(-1)}=\frac{2}{15} \frac{1}{C_{R}-1+\sqrt{C_{\nu}} S K / \varepsilon}
$$

based on the identification $\tau / K \sim \sqrt{C_{\nu}}$. This type of modeling could be particularly interesting when applied to the coefficients of the quadratically nonlinear models of Refs. 5,11 , and 12 .

Some modifications of the summation procedures used here suggest themselves. First, it is perhaps closer to the spirit of Padé approximation to substitute $\Pi$ itself into its perturbation series instead of $\tau$. The procedure would lead to fast pressure strain models which are explicit function of $\nabla U$ and $\nabla U^{T}$. Although previous experience strongly suggests the appearance of $\tau$ in the model, this unconventional procedure may deserve further consideration. A second related possibility is to apply the Padé method to the perturbation series $^{5}$ for $\tau$ directly. This also will produce a family of implicit models linear in $\tau$ and of all orders in $\nabla U$ and $\nabla U^{T}$, and to explicit models of the form (30). The lowest order such model, analogous to the LRR model, would have the form 


$$
\begin{gathered}
\overline{u_{i} u_{j}}=\frac{2}{3} K \delta_{i j}-\nu\left(\frac{\partial U_{i}}{\partial x_{j}}+\frac{\partial U_{j}}{\partial x_{i}}\right)+C_{1} \frac{K}{\varepsilon}\left({\overline{u_{i} u_{p}}}^{(0)} \frac{\partial U_{j}}{\partial x_{p}}+{\overline{u_{j} u_{p}}}^{(0)} \frac{\partial U_{p}}{\partial x_{i}}\right) \\
+C_{2} \frac{K}{\varepsilon}\left({\overline{u_{i} u_{p}}}^{(0)} \frac{\partial U_{p}}{\partial x_{j}}+{\overline{u_{j} u_{p}}}^{(0)} \frac{\partial U_{i}}{\partial x_{p}}\right)
\end{gathered}
$$

with model constants $C_{1}$ and $C_{2}$. This is actually the procedure followed by Yoshizawa ${ }^{14}$, although Yoshizawa's two-scale perturbation theory leads naturally to a transport model rather than to an algebraic model.

\section{The Diffusion Term}

The diffusion of Reynolds stress arises from the triple correlation

$$
\frac{\partial}{\partial x_{p}}\left\langle u_{i} u_{j} u_{p}\right\rangle
$$

Lowest order analysis of this term will lead to a diffusive term

$$
\frac{\partial}{\partial x_{p}} \alpha_{\tau} \nu \frac{\partial \overline{u_{i} u_{j}}}{\partial x_{p}}
$$

where $\alpha_{\tau} \sim 1.4$ in the high Reynolds number limit ${ }^{4}$, otherwise expressed, to an isotropic diffusivity for Reynolds stress $\kappa=\alpha_{\tau} \nu$.

In Ref. 22, we analyzed the diffusion of a passive scalar to second order in the $\eta$ expansion, and found, as in a similar analysis by Yoshizawa ${ }^{25}$, corrections leading to

$$
\kappa_{i j}=\kappa \delta_{i j}+\kappa^{\prime} \frac{K}{\varepsilon} \frac{\partial U_{i}}{\partial x_{j}}
$$


Unlike the isotropic diffusivity, this model permits a nonzero diffusivity $\kappa_{12}$. However, it rules out inequality of the normal diffusivities. At the next order, perturbation theory will correct Eq. (31) with terms quadratic in the velocity gradients. In this theory, unequal normal diffusivities are possible. But now the discussion in the Introduction applies again: it is necessary to sum this $\eta$-expansion. The type of Padé approximation used in Sects. I-II may lead to a diffusivity dependent on the Reynolds stresses, as in the passive scalar models of Rogers et al. ${ }^{26}$ This type of diffusivity has also been proposed by Launder, Reece and Rodi $^{6}$ for the Reynolds stresses. The details are considerably more elaborate than for the pressure correlation, and this possibility will be left for future investigation.

\section{Conclusions}

The present analysis of the Reynolds stress transport equation, based on the YakhotOrszag renormalization group and (tensorial) $\eta$-expansion summation as suggested by Yakhot et al. ${ }^{9}$, has led to a model transport equation incorporating the well-known LRR and Rotta models. The analysis gives theoretical support both to these models and to the constants sometimes used with them. More significantly, it exhibits the LRR and Rotta models as lowest order approximations, and therefore also supports their replacement with higher order nonlinear models which would be deduced by more accurate approximate summations. The consistency of the analysis with higher order effects like the unequal relaxation rates of shear and normal stresses has been discussed. 


\section{REFERENCES}

1. C. G. Speziale, S. Sarkar, and T. Gatski, "Modeling the pressure-strain correlation of turbulence - an invariant dynamical systems approach," J. Fluid Mech. to appear.

2. T.H. Shih and J.L. Lumley, "Modeling of pressure correlation terms in Reynolds stress and scalar flux equations," Rept. FDA-85-3, Sibley School of Mech. and Aero. Eng. Cornell U. (1985).

3. J. Weinstock, "Theory of the pressure-strain rate: Part II, diagonal elements" J. Fluid Mech. 116, 1 (1982).

4. V. Yakhot and S. A. Orszag, "Renormalization group analysis of turbulence I. Basic theory," J. Sci. Comp. 1, 3 (1986).

5. R. Rubinstein and J.M. Barton, "Nonlinear Reynolds stress models and the renormalization group," Phys. Fluids A 2, 1472 (1990).

6. B. E. Launder, G. Reece, and W. Rodi, "Progress in the development of a ReynoldsStress turbulence closure," J. Fluid Mech. 68537 (1975).

7. J. Rotta, "Statistical theory of non-homogeneous turbulence," Z. Phys. 129, 547 (1951).

8. A. Yoshizawa, "Statistical evaluation of the triple velocity correlation and the pressurevelocity correlation in shear turbulence," J. Phys. Soc. Jpn. 51, 2326 (1982).

9. V. Yakhot, S. Thangam, T. Gatski, S. A. Orszag, and C. G. Speziale, "Development of turbulence models for shear flows by a double expansion technique," submitted to Phys. Fluids A.

10. V. Yakhot and S. A. Orszag, "Analysis of the $\epsilon$-expansion in turbulence theory: approximate renormalization group for diffusion of a passive scalar in a random velocity field," Princeton Univ. preprint (1989).

11. A. Yoshizawa, "Statistical analysis of the deviation of the Reynolds stress from its eddy-viscosity representation," Phys. Fluids 27, 1377 (1984).

12. C. G. Speziale, "On nonlinear K-1 and K- $\epsilon$ models of turbulence," J. Fluid Mech. 178, 459 (1987).

13. A. J. M. Spencer and R. S. Rivlin, "Further results in the theory of matrix polynomials," Arch. Rational Mech. Anal. 4, 214 (1960).

14. A. Yoshizawa, "Deviation of a model Reynolds stress transport equation using a renormalization of the eddy-viscosity-type representation," preprint.

15. V. Yakhot and L. M. Smith, "The $\epsilon$-expansion for derivation of turbulence models," submitted to Phys. Fluids A.

16. S. Crow, "Viscoelastic properties of fine grained incompressible turbulence," J. Fluid Mech. 41, 81 (1968). 
17. V. Yakhot, private communication.

18. L. M. Smith and W. C. Reynolds, "A critical assessment of the Yakhot-Orszag renormalization group method for deriving turbulence models and statistics," submitted to Physics of Fluids A (1991).

19. C. G. Speziale and N. MacGiolla Mhuiris, "On the prediction of equilibrium states in homogeneous turbulence," J. Fluid Mech. 209, 591 (1989).

20. W. Rodi, "A new algebraic relation for computing the Reynolds stresses," Z. Ang. Math. Mech. 56, 219 (1976).

21. W. C. Reynolds, "Fundamentals of turbulence for turbulence modeling and simulation," AGARD Lecture Series No. 86, 1 (1987).

22. R. Rubinstein and J.M. Barton, "Renormalization group analysis of anisotropic diffusion in turbulent shear flow," Phys. Fluids A 3, 415 (1991).

23. S. G. Pope, "A more general effective-viscosity hypothesis," J. Fluid Mech. 72, 331 (1975).

24. K. Horiuti, "Higher order terms in the anisotropic representation of Reynolds stresses," Phys. Fluids A 2, 1708 (1990).

25. A. Yoshizawa, "Statistical modelling of passive scalar diffusion in turbulent shear flows," J. Fluid Mech. 195, 541 (1988).

26. M. M. Rogers, P. Mansour, and W. C. Reynolds, "An algebraic model for the turbulent flux of a passive scalar," J. Fluid Mech. 203, 77 (1989).

27. S. Tavoularis and S. C. Corrsin, "Experiments in nearly homogeneous turbulent shear flow with a uniform mean temperature gradient Part 1," J. Fluid Mech. 104, 311 (1981).

28. M. M. Rogers, P. Moin and W. Reynolds, "The structure and modeling of the hydrodynamic and passive scalar fields in homogeneous shear flows," Dept. Mech. Eng. Rep. TF-25, Stanford U. (1986).

29. S. Tavoularis and U. Karnik, "Further experiments on the evolution of turbulent stresses and scales in uniformly sheared turbulence," J. Fluid Mech. 204, 457 (1989).

30. P. Spalart, "Direct simulation of a turbulent boundary layer up to $R_{\theta}=1410$," NASA TM 89407 (1986).

31. J. Laufer, "The structure of turbulence in fully developed pipe flow," NACA Tech. Rep. 1174 (1954).

32. V. G. Harris, J. A. Graham and S. C. Corrsin, "Further experiments in nearly homogeneous turbulent shear flow," J. Fluid Mech. 81, 657 (1977). 
Public reporting burden for this collection of inlormation is estimated to average 1 hour per response, including the time for reviewing instructions, searching existing data sources, gathering and maintaining the data needed, and completing and reviewing the collection of information. Send comments regarding this burden estimate or any other aspect of this

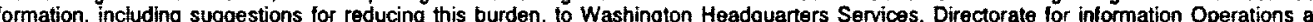
Davis Highway, Suite 1204, Arlington, VA 22202-4302, and to the Office of Management and Budget, Paperwork Reduction Project (0704-0188), Washington, DC 20503.

\begin{tabular}{|l|l|l} 
1. AGENCY USE ONLY (Leave blank) & $\begin{array}{r}\text { 2. REPORT DATE } \\
\text { March } 1992\end{array}$ & $\begin{array}{r}\text { 3. REPORT TYPE AND DATES COVERED } \\
\text { Technical Memorandum }\end{array}$ \\
\hline
\end{tabular}

4. TITLE AND SUBTITLE 5. FUNDING NUMBERS

Renormalization Group Analysis of the Reynolds Stress Transport Equation

\section{AUTHOR(S)}

WU-505-62-21

R. Rubinstein and J.M. Barton

7. PERFORMING ORGANIZATION NAME(S) AND ADDRESS(ES)

8. PERforming organization REPORT NUMBER

National Aeronautics and Space Administration

Lewis Research Center

Cleveland, Ohio 44135-3191

E- 6917

\section{SPONSORING/MONITORING AGENCY NAMES(S) AND ADDRESS(ES)}

National Aeronautics and Space Administration

Washington, D.C. 20546-0001
10. SPONSORINGMONTOAING AGENCY REPORT NUMBER

NASA TM- 105588

ICOMP-92-06; CMOTT-92-03

11. SUPPLEMENTARY NOTES

R. Rubinstein and J.M. Barton, Sverdrup Technology, Inc., Lewis Research Center Group, 2001 Aerospace Parkway, Brook Park, Ohio 44142. This work was done in cooperation with the Institute for Computational Mechanics in Propulsion and Center for Modeling of Turbulence and Transition, Lewis Research Center, Cleveland, Ohio (work funded under NASA Cooperative Agreement NCC3-233). ICOMP Program Director, Louis A. Povinelli, (216) 433-5818.

12a. DISTRIBUTION/AVAILABILITY STATEMENT $\quad$ 12b. DISTRIBUTION CODE

Unclassified - Unlimited

Subject Category 34

\section{ABSTRACT (Maximum 200 words)}

The pressure-velocity correlation and return to isotropy term in the Reynolds stress transport equation are analyzed using the Yakhot-Orszag renormalization group. The perturbation series for the relevant correlations, evaluated to lowest order in the $\varepsilon$-expansion of the Yakhot-Orszag theory, are infinite series in tensor product powers of the mean velocity gradient and its transpose. Formal lowest order Padé approximations to the sums of these series produce a fast pressure strain model of the form proposed by Launder, Reece, and Rodi, and a return to isotropy model of the form proposed by Rotta. In both cases, the model constants are computed theoretically. The predicted Reynolds stress ratios in simple shear flows are evaluated and compared with experimental data. The possibility is discussed of driving higher order nonlinear models by approximating the sums more accurately.

14. SUBJECT TERMS

Turbulence modeling

17. SECURITY CLASSIFICATION OF REPORT Unclassified

18. SECURITY CLASSIFICATION
OF THIS PAGE
Unclassified

Unclassified
19. SECURTY CLASSIFICATION OF ABSTRACT Unclassified
15. NUMBER OF PAGES 26

16. PRICE CODE

$\mathrm{A} 03$

20. LIMITATION OF ABSTRACT

Standard Form 298 (Rev. 2-89) 